\title{
Improvement in Polysomnographic Objective Sleep Quality in Adults with Pectus Excavatum After the Nuss Procedure
}

\author{
Mei-Chen Yang ${ }^{1,2}$ • Chou-Chin Lan ${ }^{1,2} \cdot$ Yao-Kuang Wu ${ }^{1,2} \cdot$ Min-Shiau Hsieh ${ }^{2,3} \cdot$ Bo-Chun Wei $^{3}$. \\ Yeung-Leung Cheng ${ }^{2,3}$ (1)
}

Published online: 13 December 2019

(C) The Author(s) 2019

\begin{abstract}
Background Patients with pectus excavatum have a poorer subjective sleep quality and quality of life than the general population. The Nuss procedure has been shown to improve these patients' quality of life, but data regarding their postoperative sleep quality are lacking. We aimed to evaluate the objective sleep quality of adults with pectus excavatum before and after the Nuss procedure.

Methods Twenty-eight participants completed this study. Epworth Sleepiness Scale (ESS) scores for daytime sleepiness, Pittsburgh Sleep Quality Index (PSQI) scores for subjective sleep quality, and overnight polysomnography for objective sleep quality were evaluated before and 6 months after the Nuss procedure.

Results Subjective sleep quality improved after the Nuss procedure. The median PSQI score decreased from 7 [interquartile range (IQR): 5; 9] to 5 (IQR: 4; 7, $p=0.029$ ); the median percentage of poor PSQI sleep quality decreased from 64.3 to $35.7 \%(p=0.048)$. The median percentage of rapid eye movement sleep significantly increased after surgery [15.6\% (IQR: $12.2 \% ; 19.8 \%$ ) vs. 20.4\% (IQR: $14.5 \% ; 24.9 \%$ ), $p=0.016]$. Sleep interruptions also improved, with the median arousal index decreasing from 9.5 (IQR: 4.8; 18.2) to 8.2 (IQR: $4.3 ; 12.1 ; p=0.045$ ). However, there was no significant change in ESS scores after surgery $(p=0.955)$.

Conclusions Pectus excavatum may be associated with poor subjective and objective sleep quality in adults, and the condition may improve after the Nuss procedure. For adults with pectus excavatum who report poor subjective sleep quality, polysomnography should be considered to assess their preoperative and postoperative sleep condition.
\end{abstract}

Yeung-Leung Cheng

ndmc0928@yahoo.com.tw

1 Division of Pulmonary Medicine, Department of Internal Medicine, Taipei Tzu Chi Hospital, Buddhist Tzu Chi Medical Foundation, New Taipei, Taiwan

2 School of Medicine, Tzu Chi University, Hualien, Taiwan

3 Division of Thoracic Surgery, Department of Surgery, Taipei Tzu Chi Hospital, Buddhist Tzu Chi Medical Foundation, No. 289, Jianguo Rd., Xindian Dist., New Taipei 23143, Taiwan

\section{Introduction}

Pectus excavatum (PEx) is the most common congenital chest deformity caused by overgrowth of costal cartilages. As the deformed chest wall worsens, the compression of the heart and lungs may become serious, resulting in clinical symptoms and affecting the patient's quality of life (QOL) [1-6].

Surgical correction is the main treatment for PEx. A minimally invasive technique for PEx repair was introduced by Dr. Donald Nuss in 1998 [7]. The Nuss procedure uses steel plate(s) under the depressed thoracic wall with the aid of a thoracoscope. The popularity of this procedure has increased rapidly $[3,8,9]$. The procedure provides 
support to the depressed thoracic wall, thereby reducing the compression on cardiopulmonary organs.

Previous studies have shown that QOL improved after the Nuss procedure [3,10-13]. However, sleep quality is an important aspect of QOL and should be included in QOL evaluations [14, 15]. The Pittsburgh Sleep Quality Index (PSQI) is widely used to evaluate subjective sleep quality, and the Epworth Sleepiness Scale (ESS) questionnaire is used to assess daytime sleepiness [16, 17]. Our team conducted a study to examine the subjective sleep quality of adult PEx patients using the PSQI questionnaire. We found that PEx may be associated with poor subjective sleep quality, which may improve after Nuss surgery [18]. To date, objective sleep quality has not been investigated in PEx adults. We aimed to evaluate objective sleep quality using polysomnography in PEx adults before and after Nuss surgery.

\section{Materials and methods}

\section{Participants}

The study was approved by the institutional review board of Taipei Tzu Chi Medical Foundation in New Taipei City, Taiwan (Protocol No.: 04-XD15-056). All adults with PEx scheduled for Nuss surgery at the Division of Thoracic Surgery in Taipei Tzu Chi Hospital from October 2015 to June 2017 were recruited. The inclusion criteria were $\geq 20$ years of age with a Haller index $\geq 3.2$ [19], no psychiatric or medical illness (heart, renal, and/or liver disease or chronic airway obstruction), and no use of psychoactive, soporific, or illegal drugs. Participation was completely voluntary, and written informed consent was obtained from each participant.

Baseline clinical characteristics, including age, gender, body height $(\mathrm{BH})$, body weight $(\mathrm{BW})$, body mass index (BMI, $\mathrm{kg} / \mathrm{m}^{2}$ ), pulmonary function evaluated by spirometry, and postoperative data, were collected. The indications for surgical repair followed the criteria proposed by Dr. Nuss [20].

The surgical techniques, which employed a bilateral thoracoscopy-assisted Nuss procedure, were performed by Cheng as previously described and demonstrated on video [18]. All patients were interviewed at baseline, with a follow-up about 6 months after Nuss surgery. The patients were encouraged to return to normal daily activity and normal exercise 3 months after the operation.

A total of 108 patients were scheduled for a Nuss procedure during the study interval. Sixty-eight patients met the inclusion criteria and were invited to participate in the study; 32 patients agreed. These patients underwent the Nuss procedure as scheduled without perioperative complications and recovered uneventfully during hospitalization. These patients were asked to complete the PSQI questionnaire, ESS questionnaire, and overnight polysomnography evaluations at baseline and about 6 months after the Nuss procedure.

\section{Assessment of daytime sleepiness}

Daytime sleepiness was subjectively evaluated using the Chinese version of the ESS questionnaire [17].

\section{Assessment of subjective sleep quality}

The validated Chinese version of the PSQI questionnaire was used to assess subjective sleep quality [16, 21]. The scale consists of 19 items with 7 components: sleep quality (PSQI-1), sleep latency (PSQI-2), sleep duration (PSQI-3), sleep efficiency (PSQI-4), sleep disturbance (PSQI-5), frequency of sleeping-medication use (PSQI-6), and daytime functional impairment (PSQI-7). Each PSQI item was rated from 0-3 ( $0=$ no difficulty, $3=$ extreme difficulty). The PSQI sleep quality was calculated using total PSQI scores, which ranged from 0 to 21 (higher total PSQI scores indicated poorer sleep quality). Good sleep quality was defined as total PSQI scores $\leq 5$, while poor sleep quality was defined as total PSQI scores $\geq 6$.

\section{Assessment of objective sleep quality}

Standard overnight polysomnography was performed at the sleep center. During polysomnography, electroencephalography (EEG), electrooculography, chin and bilateral anterior tibialis surface electromyography, electrocardiography, and assessments of airflow through the nose and mouth (registered by a thermistor and nasal pressure sensor), thoracoabdominal movements (registered by respiratory inductive plethysmography), position (by a sensor on the respiratory inductive plethysmography unit), snoring, and oxygen saturation (by pulse oximetry) were performed. The polysomnography lasted for at least $6 \mathrm{~h}$ and was analyzed via manual scoring of every $30 \mathrm{~s}$ epoch by trained sleep technicians and reviewed by a sleep specialist. Sleep stage was manually scored using the standard criteria proposed by Rechtschaffen and Kales [22]. An apnea event was defined as an $80-100 \%$ reduction in airflow lasting for at least $10 \mathrm{~s}$. A hypopnea event was defined as a reduction in airflow of at least $50 \%$ for at least $10 \mathrm{~s}$ or of at least $30 \%$ for at least $10 \mathrm{~s}$ in comparison with the baseline and associated with at least 3\% oxygen desaturation or an EEG arousal. The apnea/hypopnea index (AHI) was calculated as the ratio of the number of apnea and hypopnea events per hour. The desaturation index (DI) was defined as $>3 \%$ oxygen desaturation per hour. The arousal index $(\mathrm{AI})$ was defined in terms of the number of arousal 
episodes per hour [23]. Sleep efficiency was defined as the fraction of total sleep time to total recording time. Sleep latency was defined as the time from lights off to the first identifiable sleep stage. Rapid eye movement (REM) latency was defined as the time from the first identifiable sleep stage to the first REM sleep.

\section{Statistical analysis}

Continuous variables were presented as the median and interquartile range (IQR: 25 th and 75th percentiles) and all displayed non-normal distribution. Categorical variables were summarized as a count (\%). Continuous variables before and after the Nuss procedure were compared using the Wilcoxon signed-rank test. Categorical variables before and after the Nuss procedure were compared using the Fisher exact test. $p$ values $<0.05$ were considered statistically significant. Statistical analyses were performed using SPSS software (version 24; SPSS; Chicago, IL, USA).

\section{Results}

Among the 32 participants, four withdrew from the study after operation (one studied in Germany, one worked in the Republic of China, one was excluded due to a surgical complication [flipping of the bar found one month after surgery], and one was lost to follow-up). Thus, 28 patients were included in the final analysis. The mean hospital stay was $6.5 \pm 1.1$ days. The mean postoperative follow-up period was 16.5 months (range: 12-20 months).

Table 1 shows the demographic characteristics of the 28 participants. The median age was 24.0 years (IQR: 22.0; 27.5). The majority of the patients were male $(25 / 28$ patients: $89.3 \%$ ). The median BMI was $20.2 \mathrm{~kg} / \mathrm{m}^{2}$ (IQR: $18.9 ; 21.9)$. None of the patients smoked cigarettes. The median Haller index was 3.6 (IQR: 3.3; 4.3). The median forced expiratory volume in $1 \mathrm{~s} /$ forced vital capacity (FEV1/FVC) was $87.5 \%$ (IQR: 83.3; 91.0), with the median FEV1 being $83.5 \%$ (IQR: 73.5; 91.3) and median FVC being $80 \%$ (IQR: 69.5 ; 91.3). Only two patients $(7.1 \%)$ had restrictive ventilatory impairment. The median postoperative interval for performing the overnight polysomnography examination was 6.54 months (range: 5.8-10.6 months).

Table 2 shows the ESS scores, PSQI, and polysomnography results before and after the Nuss procedure. The median ESS scores showed no significant difference after surgery (6.5 vs. 6.0, $p=0.955)$. Total PSQI scores decreased from 7 to $5(p=0.029)$ (Fig. 1a). The median percentage of poor PSQI sleep quality significantly decreased from 64.3 to $35.7 \%(p=0.048)$. There was no
Table 1 Demographic characteristics of 28 adult patients with pectus excavatum

\begin{tabular}{lc}
\hline Characteristics & Cases $(n=28)$ \\
\hline Age, years (IQR) & $24.0(22.0 ; 27.5)$ \\
Gender & $25(89.3 \%)$ \\
Male, $n(\%)$ & $3(10.7 \%)$ \\
Female, $n(\%)$ & $173(167 ; 177.8)$ \\
Body height, cm (IQR) & $62.5(55.0 ; 65.8)$ \\
Body weight, kg (IQR) & $20.2(18.9 ; 21.9)$ \\
Body mass index, kg/m ${ }^{2}$ (IQR) & $3.6(3.3 ; 4.3)$ \\
Haller index (IQR) & \\
Pulmonary function rest & $80.0(69.5 ; 91.3)$ \\
FVC, \% of predicted value (IQR) & $83.5(73.5 ; 91.3)$ \\
FEV1, \% of predicted value (IQR) & $87.5(83.3 ; 91.0)$ \\
FEV1/FVC, \% (IQR) & $88.5(84.0 ; 96.0)$ \\
TLC, \% of predicted value (IQR) & $2(7.1 \%)$ \\
Restrictive lung defect, $n(\%)$ & \\
\hline
\end{tabular}

Continuous data (age, body height, body weight, body mass index, Haller index) are shown as the median and interquartile range, and categorical data (gender) are shown as a count (\%)

$I Q R$ interquartile range, $F V C$ forced vital capacity, FEVI forced expiratory volume in $1 \mathrm{~s}, T L C$ total lung capacity

statistical difference in subjective PSQI sleep efficiency after surgery $(91.3 \%$ vs. $90.5 \%, \quad p=0.988)$. For polysomnography results, the objective sleep efficiency showed no difference before and after the Nuss procedure (77\% vs. $78.6 \%, p=0.284)$. $N 1$ and $N 2$ sleep did not differ after surgery $(8.1 \%$ vs. $9.5 \%, p=0.716$ and $56.4 \%$ vs. $59.9 \%, p=0.829$, respectively). Non-rapid eye movement (NREM) sleep significantly decreased after surgery $(84.4 \%$ vs. $79.6 \%, p=0.015$ ), which was mainly due to the decrease in $N 3$ sleep $(18.1 \%$ vs. $12.7 \%, p=0.019)$. REM sleep significantly increased after the Nuss procedure ( $15.6 \%$ vs. $20.4 \%, p=0.016$ ) (Figs. $1 \mathrm{~b}$ and 2 ). The AI significantly improved after the Nuss procedure (9.5 vs. $8.2, p=0.045)$. There were no differences in sleep latency, REM latency, mean and nadir $\mathrm{SaO}_{2}$, mean heart rate, DI, or AHI after the Nuss procedure.

\section{Discussion}

Our results showed that adults with PEx may be associated with both poor subjective and objective sleep quality (less REM sleep and more interrupted sleep), which could be improved after the Nuss procedure. To our knowledge, this is the first study using polysomnography to evaluate objective sleep quality in PEx patients. Although our study sample was small, the gender distribution and demographic characteristics of our patients were similar to those in 
Table 2 Comparison of the subjective sleep quality, daytime sleepiness, and objective sleep quality of 28 adults with pectus excavatum before and after Nuss procedure

\begin{tabular}{|c|c|c|c|}
\hline Characteristics & Before Nuss procedure & After Nuss procedure & $p$ value \\
\hline Daytime sleepiness (ESS) (IQR) & $6.5(4.3 ; 8.8)$ & $6(4 ; 10.5)$ & 0.955 \\
\hline \multicolumn{4}{|l|}{ Subjective sleep quality (PSQI) } \\
\hline Total PSQI score & $7(5 ; 9)$ & $5(4 ; 7)$ & $0.029 *$ \\
\hline PSQI sleep quality & & & $0.048 *$ \\
\hline Good sleep quality (PSQI $\leqq 5), n(\%)$ & $10(35.7 \%)$ & $18(64.3 \%)$ & \\
\hline Poor sleep quality (PSQI $\geqq 6), n(\%)$ & $18(64.3 \%)$ & $10(35.7 \%)$ & \\
\hline Sleep efficiency, \% (IQR) & $91.3(85.7 ; 100)$ & $90.5(86.0 ; 100)$ & 0.988 \\
\hline \multicolumn{4}{|c|}{ Objective sleep quality (polysomnography variables) } \\
\hline Sleep efficiency, \% (IQR) & $77.0(65.6 ; 83.9)$ & $78.6(72.5 ; 87.2)$ & 0.284 \\
\hline$N 1$ stage, $\%(\mathrm{IQR})$ & $8.1(5.5 ; 14.6)$ & $9.5(6.6 ; 12.2)$ & 0.716 \\
\hline$N 2$ stage, \% (IQR) & $56.4(51.7 ; 63)$ & $59.9(48 ; 65.0)$ & 0.829 \\
\hline$N 3$ stage, \% (IQR) & $18.1(10.7 ; 22.1)$ & $12.7(7.3 ; 17.2)$ & $0.019 *$ \\
\hline NREM stage $(N 1+N 2+N 3), \%(\mathrm{IQR})$ & $84.4(80.2 ; 87.8)$ & $79.6(75.1 ; 85.4)$ & $0.015^{*}$ \\
\hline REM stage, \% (IQR) & $15.6(12.2 ; 19.8)$ & $20.4(14.5 ; 24.9)$ & $0.016^{*}$ \\
\hline Sleep latency, $\min (\mathrm{IQR})$ & $42.5(23.0 ; 76.3)$ & $42.5(21.5 ; 88.1)$ & 0.767 \\
\hline REM latency, min (IQR) & $82.5(66.8 ; 148.0)$ & $87.3(69.4 ; 119.9)$ & 0.936 \\
\hline Mean $\mathrm{SaO}_{2}, \%$ (IQR) & $95.0(95.0 ; 96.0)$ & $95.0(95.0 ; 96.8)$ & 0.134 \\
\hline Nadir $\mathrm{SaO}_{2}, \%$ (IQR) & $91.0(89.0 ; 92.0)$ & $91.5(88.0 ; 93.0)$ & 0.767 \\
\hline Mean heart rate, beats/min (IQR) & $64.0(55.8 ; 69.5)$ & $65.0(60.0 ; 68.5)$ & 0.213 \\
\hline Desaturation index, events/h (IQR) & $1.9(0.4 ; 2.9)$ & $0.7(0.2 ; 3.1)$ & 0.143 \\
\hline Apnea-hypopnea index, events/h (IQR) & $2.8(0.9 ; 6.4)$ & $2.1(0.3 ; 4.9)$ & 0.194 \\
\hline Arousal index, events/h (IQR) & $9.5(4.8 ; 18.2)$ & $8.2(4.3 ; 12.1)$ & $0.045^{*}$ \\
\hline
\end{tabular}

Continuous variables are shown as the median (interquartile range) and were analyzed using the Wilcoxon signed-rank tests. Categorical variables are shown as the count $(\%)$ and were analyzed using Fisher's exact test. $p<0.05$ indicated significant difference before and after the Nuss procedure

ESS Epworth sleepiness scale, IQR interquartile range, PSQI Pittsburg sleep quality index, NREM non-rapid eye movement, $R E M$ rapid eye movement, $\mathrm{SaO}_{2}$ oxygen saturation

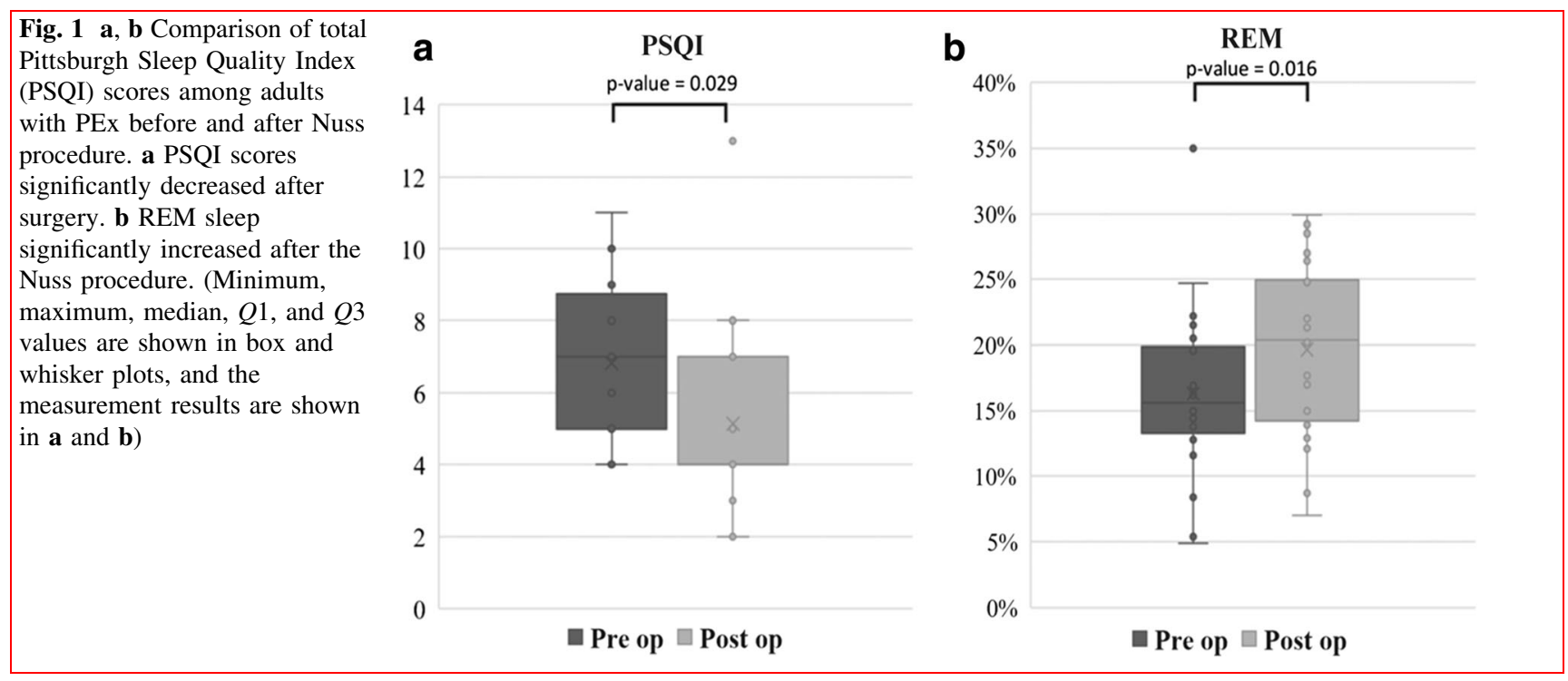


Fig. 2 Polysomnography results of a 25 -year-old male with pectus excavatum (body height $168 \mathrm{~cm}$; body weight $56 \mathrm{~kg}$, body mass index 19.8) before (upper part) and after Nuss procedure (lower part). Rapid eye movement sleep increased after surgery $(14.2 \%$ vs. $20.0 \%)$. Sleep fragmentation improved after surgery
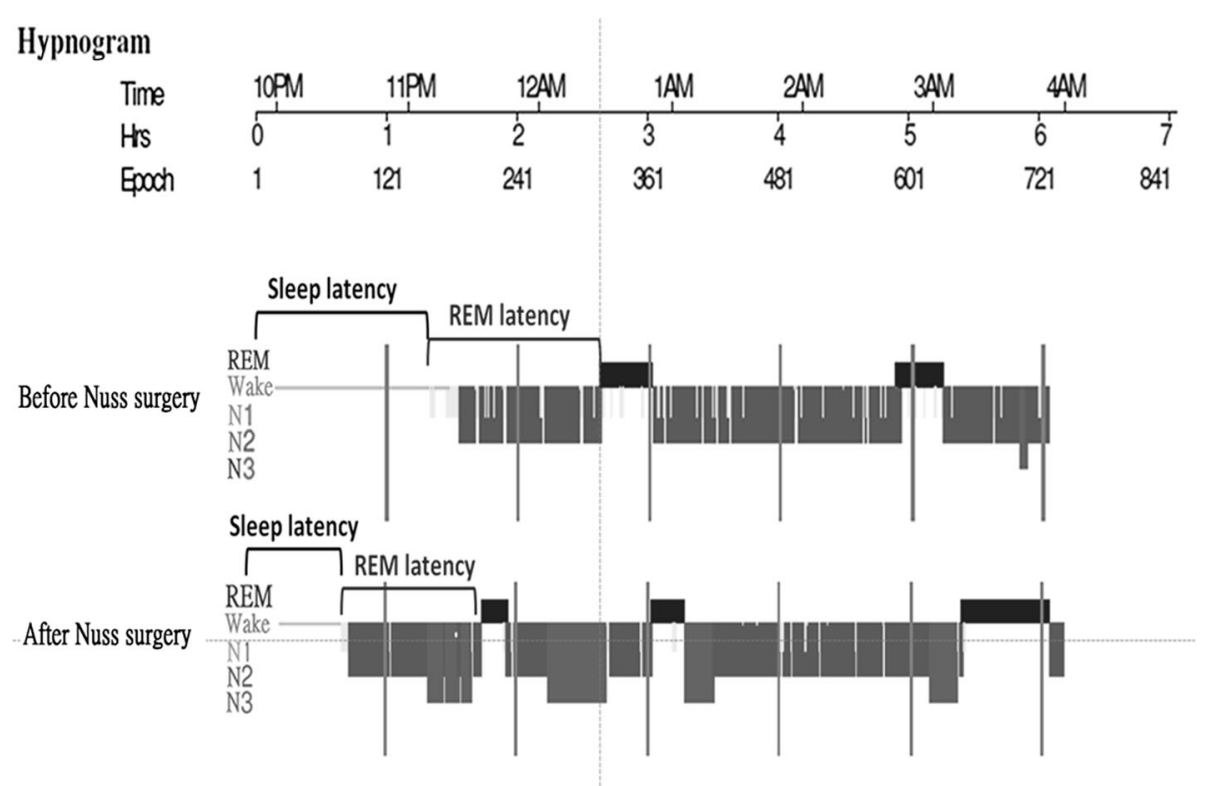

previous studies [24, 25]. As such, our sample may represent the actual situation of PEx patients; our results are valuable because they provide an additional surgical indication for the Nuss procedure in PEx adults.

The QOL of PEx patients was often evaluated in previous investigations. Our previous study showed the subjective sleep quality of adults with PEx was poorer than that of the general population and could be improved after the Nuss procedure [18]. In this study, we obtained similar results. The PSQI questionnaire is a relatively quick and simple way to evaluate subjective sleep quality. We believe the PSQI questionnaire is valuable for evaluating sleep quality as a part of the QOL of adults with PEx. Shen et al. found PSQI score positively correlated with age in hemodialysis patients (elderly patients had higher PSQI scores, poorer subjective sleep quality) [26]. Our patients were all aged over 20 years. A future study focusing on individuals younger than 20 years of age is needed.

In 1981, Guilleminault et al. studied the polysomnography findings of five patients with severe restrictive lung disease (severe kyphoscoliosis-related musculoskeletal deformities) and found these patients often complained of disrupted sleep with frequent arousal because of sleep-associated hypoxemia and hypercapnia [27]. We also observed interrupted sleep in our patients; however, we did not observe hypoxemia or hypercapnia, and the pulmonary function tests yielded relatively normal results. However, the interrupted sleep improved after the Nuss procedure, suggesting there might be other factors beyond hypoxemia and hypercapnia disrupting their sleep. The physiological thoracic wall compression caused by PEx or its psychological impact might have a negative impact on sleep continuity. Further studies are needed to address this issue.

Patients with restrictive lung disease also had excessive daytime sleepiness, as thoracic cage deformity alters chest wall mechanics [28]. In our study, the ESS scores did not change after operation because the ESS questionnaire only assessed patients' attention ability while performing daily activities such as watching television, talking with others, or waiting for a traffic light. The ESS questionnaire is used clinically to evaluate the severity of sleep-disordered breathing (SDB) and might be not suitable to evaluate the degree of fatigue in dynamic daily activities such as housework or exercise. Other methods, such as the 6-minute walking test for submaximal exercise capacity or a comprehensive cardiopulmonary test for maximal exercise capacity, might be more useful for daytime fatigue evaluation in PEx patients.

Although polysomnography remains the gold standard in sleep investigations, there is some debate regarding the use of single-night recordings because of the first-night effect and the night-to-night variability in AHI [29]. The normal range of sleep efficiency is $85-95 \%$, and the normal ranges of $N 1, N 2, N 3$, and REM sleep for young adults are $2-5 \%, 45-55 \%, 13-23 \%$, and $20-25 \%$, respectively [30]. The first-night effect, mainly characterized by lower sleep efficiency, longer sleep and REM latency, more $N 2$ sleep, and less $N 3$ and REM sleep during the first night, has been reported on the first two consecutive nights of polysomnography rest [29, 31]. In the present study, the patients received the second polysomnography examination more than 6 months after the first. In our data, only 
REM sleep showed significant improvement after operation. Even so, in previous studies, elderly patients, especially those aged more than 40 years, showed a greater first-night effect $[31,32]$. Most of our patients were aged 20-30 years. Therefore, our results were not entirely influenced by the first-night effect.

There are many factors affecting sleep quality, including age, BMI, upper respiratory tract variation, brain injury, alcohol, and drug use. The median age of our patients was 24 years, and the median BMI was 20.2. None of the patients had other special medical circumstances or histories of long-term medication use, making this study population at low risk of poor sleep quality. The subjective PSQI questionnaire and the polysomnography in this study showed meaningful improvement after surgery. As such, it can be preliminarily conceived that the chest depression itself might be an important factor affecting the sleep quality of PEx patients. Therefore, the improvements in REM sleep and sleep continuity in our patients after the Nuss procedure might be attributed to the effects of surgical correction of the pectus deformity. The median AI in our patients was 9.5 per hour, similar to that in the general population [23]; the AI improved after the Nuss procedure, indicating that correction of the pectus deformity may have a beneficial effect on sleep continuity. Since one of the characteristics of SDB is sleep fragmentation with increasing AI, we believed that the improvement in AI of our patients might be the result of the operation.

Indications for the Nuss procedure for PEx include two or more of the following criteria: (1) cardiopulmonary compression symptoms, (2) Haller index $>3.2$, (3) restrictive/obstructive lung impairment, (4) mitral valve prolapse, and (5) cardiac compression [7, 18]. The indications for surgery in our patients were based on these criteria. Our present study and previous study [18] revealed that subjective and objective sleep quality in PEx adults was poor and could be improved after the Nuss procedure. In terms of clinical application, if an adult with PEx reports poor subjective sleep quality, the practitioner might consider polysomnography to further evaluate their objective sleep quality, to exclude other sleep-disordered breathing, and to evaluate the effect of the Nuss procedure.

There were several limitations to our study. First, the sample size was small and might not represent the entire population. Second, we only focused on PEx adults. There might be age-related differences between adults and children with PEx. Third, we did not include normal Taiwanese subjects for comparison. This approach may have overlooked racial differences. Larger multicenter (even international) studies are warranted.

In conclusion, we found PEx in adults may be associated with poor subjective and objective sleep quality, which could be improved after the Nuss procedure. For adults who report poor subjective sleep quality, polysomnography should be considered as an auxiliary method to evaluate their sleep quality and surgical outcome.

Funding The study was funded by Grants from the Taipei Tzu Chi Hospital, Buddhist Tzu Chi Medical Foundation (Grant Numbers TCRD-TPE-106-RT-1 and TCRD-Taipei-108-28).

\section{Compliance with ethical standards}

Conflict of interest The authors declare that they have no conflict of interest.

Ethical approval The Institutional Review Board of Taipei Tzu Chi Hospital, Buddhist Tzu Chi Medical Foundation approved this study on August 20, 2015 (Protocol No: 04-XD15-056).

Informed consent Informed consent was obtained from all individual participants included in the study.

Open Access This article is licensed under a Creative Commons Attribution 4.0 International License, which permits use, sharing, adaptation, distribution and reproduction in any medium or format, as long as you give appropriate credit to the original author(s) and the source, provide a link to the Creative Commons licence, and indicate if changes were made. The images or other third party material in this article are included in the articleã s Creative Commons licence, unless indicated otherwise in a credit line to the material. If material is not included in the articleã s Creative Commons licence and your intended use is not permitted by statutory regulation or exceeds the permitted use, you will need to obtain permission directly from the copyright holder. To view a copy of this licence, visit http://creati vecommons.org/licenses/by/4.0/.

\section{References}

1. Ji Y, Liu W, Chen S et al (2011) Assessment of psychosocial functioning and its risk factors in children with pectus excavatum. Health Qual Life Outcomes 9:28

2. Koumbourlis AC (2009) Pectus excavatum: pathophysiology and clinical characteristics. Paediatr Respir Rev 10:3-6

3. Hanna WC, Ko MA, Blitz M et al (2013) Thoracoscopic Nuss procedure for young adults with pectus excavatum: excellent midterm results and patient satisfaction. Ann Thorac Surg 96:1033-1038

4. Kragten HA, Siebenga J, Hoppener PF et al (2011) Symptomatic pectus excavatum in seniors (SPES): a cardiovascular problem? A prospective cardiological study of 42 senior patients with a symptomatic pectus excavatum. Neth Heart J 19:73-78

5. Kuru P, Bostanci K, Ermerak NO et al (2015) Quality of life improves after minimally invasive repair of pectus excavatum. Asian Cardiovasc Thorac Ann 23:302-307

6. Koumbourlis AC (2015) Pectus deformities and their impact on pulmonary physiology. Paediatr Respir Rev 16:18-24

7. Nuss D, Kelly RE, Croitoru DP et al (1998) A 10-year review of a minimally invasive technique for the correction of pectus excavatum. J Pediatr Surg 33:545-552

8. Neviere R, Montaigne D, Benhamed L et al (2011) Cardiopulmonary response following surgical repair of pectus excavatum in adult patients. Eur J Cardiothorac Surg 40:e77-e82 
9. Yeh SW, Yang MC, Chang YW et al (2019) Improved dynamics of thoracic cage and exercise capacity after Nuss procedure for pectus excavatum. Thorac Cardiovasc Surg. https://doi.org/10. 1055/s-0039-1683384 (Epub ahead of print)

10. Zuidema WP, Oosterhuis JWA, Zijp GW et al (2018) Early consequences of pectus excavatum surgery on self-esteem and general quality of life. World J Surg 42:2502-2506. https://doi. org/10.1007/s00268-018-4526-9v

11. Hoksch B, Kocher G, Vollmar P et al (2016) Nuss procedure for pectus excavatum in adults: long-term results in a prospective observational study. Eur J Cardiothorac Surg 50:934-939

12. Casamassima MGS, Gause C, Goldstein SD et al (2016) Patient satisfaction after minimally invasive repair of pectus excavatum in adults: long-term results of Nuss procedure in adults. Ann Thorac Surg 101:1338-1345

13. Obermeyer RJ, Cohen NS, Jaroszewski DE (2018) The physiologic impact of pectus excavatum repair. Semin Pediatr Surg 27:127-132

14. Ferrell BR, Grant M, Funk B et al (1996) Quality of life in breast cancer. Cancer Pract 4:331-340

15. Chopra I, Kamal KM (2012) A systematic review of quality of life instruments in long-term breast cancer survivors. Health Qual Life Outcomes 10:14

16. Jenkinson C, Coulter A, Bruster S et al (2002) The coronary heart disease in-patient experience questionnaire (I-PEQ (CHD)): results from the survey of National Health Service patients. Qual Life Res 11:721-727

17. Buysse DJ, Reynolds CF, Monk TH et al (1989) The Pittsburgh sleep quality index: a new instrument for psychiatric practice and research. Psychiatry Res 28:193-213

18. Cheng YL, Lan CC, Wu YK et al (2019) Poorer sleep quality among adult patients with pectus excavatum in Taiwan: a pilot study. J Thorac Cardiovasc Surg 157:769.e1-780.e1

19. Bimler HP (1985) Bimler therapy, part 1: Bimler cephalometric analysis. J Clin Orthod 19:501-523

20. Nuss D, Kelly RE (2010) Indications and technique of Nuss procedure for pectus excavatum. Thorac Surg Clin 20:583-597

21. Chan SF, Chen TH, Liao YM et al (2012) Development and preliminary validation of the Chinese version of the Sleep-Associated Monitoring Index. Int J Nurs Stud 49:54-64
22. Rechtschaffen A, Kales A, University of California et al (1968) In: Rechtschaffen A, Kales A (eds) A manual of standardized terminology, techniques and scoring system for sleep stages of human subjects, National Institute of Neurological Diseases and Blindness, Neurological Information Network, United States

23. Boselli M, Parrino L, Smerieri A et al (1998) Effect of age on EEG arousals in normal sleep. Sleep 21:361-367

24. Fonkalsrud EW (2003) Current management of pectus excavatum. World J Surg 27:502-508. https://doi.org/10.1007/s00268003-7025-5

25. Kelly RE Jr, Shamberger RC, Mellins RB et al (2007) Prospective multicenter study of surgical correction of pectus excavatum: design, perioperative complications, pain, and baseline pulmonary function facilitated by internet-based data collection. J Am Coll Surg 205:205-216

26. Shen Q, Huang X, Luo Z et al (2016) Sleep quality, daytime sleepiness and health-related quality-of-life in maintenance haemodialysis patients. J Int Med Res 44:698-709

27. Guilleminault C, Kurland G, Winkle R et al (1981) Severe kyphoscoliosis, breathing, and sleep: the "Quasimodo" syndrome during sleep. Chest 79:626-630

28. Won CH, Kryger M (2014) Sleep in patients with restrictive lung disease. Clin Chest Med 35:505-512

29. Scholle S, Wiater A, Scholle HC (2011) Normative values of polysomnographic parameters in childhood and adolescence: cardiorespiratory parameters. Sleep Med 12:988-996

30. Le Bon O, Staner L, Hoffmann G et al (2001) The first-night effect may last more than one night. J Psychiatr Res 35:165-172

31. Webb WB, Campbell SS (1979) The first night effect revisited with age as a variable. Waking Sleeping 3:319-324

32. Ma J, Zhang C, Zhang J et al (2011) Prospective study of first night effect on 2-night polysomnographic parameters in adult Chinese snorers with suspected obstructive sleep apnea hypopnea syndrome. Chin Med J (Eng) 124:4127-4131

Publisher's Note Springer Nature remains neutral with regard to jurisdictional claims in published maps and institutional affiliations. 